\title{
La cooperación como proceso: construcción de la etnografía institucional en Bangladesh
}

\author{
DAVID LEWIS*
}

\begin{abstract}
This paper presents a research into the relationships between the agencies participating in developing projects, taking as a case study an acuaculture project in Bangladesh. The roots of the present case study, can be traced back to research undertaken during 1990-92 by the Overseas Development Institute (ODI), which investigated government and nongovernmental organisation (NGO) linkages during efforts to promote technical change in the agricultural sector using case studies collected. in Asia, Africa and Latin America. The research concluded that while collaboration between NGOs and government agencies was certainly taking place and in many cases generating potentially useful new approaches and insights, there was no straightforward 'functional' division of agency roles. On the other hand, the social, political and historical contextual factors in different countries were crucial determinants of link-age effectiveness.
\end{abstract}

Keywords: NGO, development projects, acuaculture, inter-agency partnership.

\section{Resumen}

El texto presenta una investigación acerca de las relaciones entre las diferentes organizaciones que participan en los proyectos de desarrollo, tomando como caso de estudio un proyecto de acuacultura en Bangladesh. El origen de este trabajo es la investigación llevada a cabo entre 1990 y 1992 por el Instituto del Desarrollo en el Exterior (Overseas Development Institute, ODI), mediante la cual se investigaron los vínculos de las organizaciones gubernamentales y no gubernamentales (ONG) durante los esfuerzos para promover el cambio tecnológico en el sector agrícola, usando estudios de caso de Asia, África y Latinoamérica. La investigación concluye que si bien la colaboración entre las ONG y las agencias gubernamentales ciertamente estaba teniendo lugar y en muchos casos generaba nuevos enfoques e ideas potencialmente útiles, no existía una división 'funcional' verdadera de los roles de las organizaciones. Por otro lado, el contexto social, político e histórico en los diferentes países resulta ser un determinante crucial para la efectividad de los vínculos establecidos.

Palabras clave: ONG, proyectos de desarrollo, acuacultura, cooperación interorganizacional.

* Centro para la Organización Voluntaria, London School of Economics and Political Science. Correo-e: D.J.Lewis@lse.ac.uk 


\section{Introducción ${ }^{1}$}

En este artículo se presenta una investigación acerca de las relaciones en proyectos interorganizacionales. En la metodología adoptada durante esta investigación resultó central adoptar una perspectiva procesual de los proyectos, lo que generó un conjunto de problemáticas e ideas distintivas. En un nivel conceptual, las ideas acerca de los procesos también contribuyeron a ampliar nuestra comprensión de la naturaleza de la cooperación entre organizaciones durante los proyectos. Las raíces del presente trabajo pueden encontrarse en la investigación llevada a cabo entre 1990 y 1992 por el Instituto del Desarrollo en el Exterior (Overseas Development Institute, ODI), en la que se analizaron los vínculos de las organizaciones gubernamentales y no gubernamentales (ONG) durante los esfuerzos para promover el cambio tecnológico en el sector agrícola, usando estudios de caso de Asia, África y Latinoamérica. La investigación concluyó que si bien la colaboración entre las ONG y las agencias gubernamentales ciertamente estaba teniendo lugar, y en muchos casos generaba nuevos enfoques e ideas potencialmente útiles, no existía una división funcional efectiva de los roles de las organizaciones, y el contexto social, político e histórico en los diferentes países, lo que resultaba un elemento crucial para la efectividad de los vínculos (Farrington y Bebbington, 1993).

La vertiente sudasiática de la investigación, documentada por Farrington y Lewis (1993), atrajo la atención de uno de los grupos consultivos de investigación agrícola internacional: el Centro Internacional para el Manejo de los Recursos Acuáticos Vivientes (ICLARM, por sus siglas en inglés), que decidió desarrollar con el ODI un proyecto de investigación para estudiar los vínculos interorganizacionales en la acuacultura. Con el apoyo de los fondos restringidos de la entonces Administración de Desarrollo en el Exterior (ODA, por sus siglas en inglés), se diseñó un proyecto conjunto para iniciar la investigación del oDI usando como caso de estudio la participación del ICLARM en la investigación y extensión de la acuacultura en Bangladesh. Al mismo tiempo, el ICLARM buscaba desarrollar una serie de vínculos institucio-

\footnotetext{
${ }^{1}$ Esta investigación se llevó a cabo mientras el autor se encontraba trabajando como investigador asociado en el Overseas Development Institute (ODI). El artículo fue publicado originalmente en inglés en Mosse, D., J. Farrington y A. Rew (eds.) (1998), Development as process: concepts and methods for working with complexity, Routledge, London. Traducción de Alma Mancilla.
} 
nales o colaboraciones, de los tipos analizados en el proyecto de investigación de ODI tanto con el gobierno de Bangladesh como con diversas ONG.

Los objetivos de este proyecto de investigación conjunto (al que se haremos referencia en este documento como investigación ODI) eran de dos tipos:

- El objetivo primario era sugerir un arreglo institucional a través del cual pudieran crearse y sostenerse los mecanismos para promover la investigación y extensión efectivas de la acuacultura;

- El objetivo secundario era documentar el proyecto interorganizacional de acuacultura del ICLARM para obtener enseñanzas generales y desarrollar una guía para la planeación de futuros proyectos.

Antes de entrar a la discusión del proyecto y de la investigación referida, es necesario hacer algunas aclaraciones introductorias acerca de la acuacultura en Bangladesh. A pesar de los notables incrementos en la producción agrícola en años recientes -de manera que el país se aproxima actualmente a la autosuficiencia de granos-, el constante crecimiento poblacional ha ejercido una enorme presión sobre los recursos naturales. En ausencia de nuevas tierras cultivables y ante las limitadas oportunidades para intensificar aún más la producción agrícola, la acuacultura se ha convertido en una importante estrategia de desarrollo, puesto que existe la percepción generalizada de que Bangladesh posee una riqueza de recursos acuáticos subutilizados o inutilizados (Lewis, Wood y Gregory, 1996). ${ }^{2}$

\section{El proyecto ICLARM}

El ICLARM ha estado involucrado en años recientes en una serie vinculada y consecutiva de proyectos cortos de acuacultura con el gobierno de Bangladesh, financiados en gran parte por USAID (ayuda internacional de EEUU). El proyecto actual pretende desarrollar y proporcionar tecnologías de acuacultura de bajo costo y baja inversión, principalmente por medio de la difusión detallada de técnicas adecuadas para el manejo de las lagunas, incluyendo las densidades de colonias de peces, el régimen de alimentación, la preparación de los estanques y las mezclas adecuadas de

${ }^{2}$ La pesca de captura, a pesar de su potencial, ha recibido una mucho menor atención por parte de los investigadores y de las organizaciones para el desarrollo. 
especies que las familias de bajos ingresos puedan emplear fácilmente como parte de sus carteras de actividades productivas. Un componente estructural clave del proyecto es la compleja estructura de la cooperación institucional entre las agencias gubernamentales, las ONG y los investigadores internacionales. Se está capacitando a los trabajadores de extensión del gobierno y al personal de campo de las ONG, quienes transmiten esta capacitación a los grupos campesinos que buscan desarrollar la acuacultura como parte de una serie de actividades productivas apoyadas por créditos otorgados por ONG. Las mujeres que tienen muy poca o ninguna tierra forman el grupo objetivo de muchas ONG en Bangladesh.

Los orígenes del proyecto ICLARM se encuentran en los vínculos informales entre algunos integrantes del personal de campo del Comité para el Avance Rural de Bangladesh (BRAC, por sus siglas en inglés) y ciertos miembros del Instituto de Investigación de la Pesca (FRI por sus siglas en inglés); vínculos que se establecieron a finales de la década de los años 80 , cuando BRAC empezaba su programa de acuacultura y necesitaba cierto apoyo técnico. El ICLARM ya estaba entonces en contacto con el FRI. Al mismo tiempo, USAID estaba buscando formas de mejorar la efectividad de su trabajo para fortalecer los institutos de investigación agrícola nacional (incluyendo al FRI) en Bangladesh. En 1991 se estableció un taller entre todas estas agencias y 31 oNG, y nació así la idea de trabajar para involucrar a las ONG en el más amplio esfuerzo de extensión de la acuacultura en Bangladesh. ${ }^{3}$ Lo que surgió como resultado de esto fue un proyecto interorganizacional titulado: "Transferencia tecnológica y retroalimentación a través de ONG" que fue financiado por USAID. La Fase I se llevó a cabo en 1992-93 y la Fase II continuó en 1993-94 aunque se extendió, debido a retrasos, hasta 1995. Este proyecto, que denominaremos el proyecto ICLARM, es el sujeto del presente artículo.

El proyecto ICLARM involucra a agencias gubernamentales y a ONG de Bangladesh, y fue diseñado para fortalecer la capacidad de investigación en acuacultura y la sensibilidad hacia las demandas de los campesinos, así como la capacidad del sistema mayor, que actualmente abarca tanto al gobierno como a las ONG, como agente de extensión. Hay tres diferentes agencias gubernamentales participando en el proyecto ICLARM. El FRI es el cuerpo de investigación del sector público responsable de la acuacultura y

${ }^{3}$ Dr. M.V. Gupta, antiguo Investigador Decano en Acuacultura, IClARM, Dhaka, comunicación personal. 
tiene su sede en Mymensingh. El FRI es una institución de investigación relativamente nueva que no cuenta con acceso a los recursos adecuados y con un espíritu de equipo relativamente bajo, pero fue considerada por el ICLARM como poseedora del potencial para hacer una contribución a las tecnologías relevantes para el desarrollo, si se le proporcionaba el financiamiento adecuado por parte de USAID y el soporte técnico por parte del ICLARM.

El Departamento de Pesca (DF, por sus siglas en inglés) controla este servicio en el ámbito nacional, pero también carece de recursos y personal suficientes, y cuenta sólo con un Oficial de Extensión Pesquera en cada Thana, la unidad local de gobierno, que en algunas zonas puede tener hasta un millón de habitantes. El proyecto pretende involucrar a trabajadores de campo de ONG en una relación de cooperación con el personal de DF, aunque éste tiene una orientación más dirigida hacia los objetivos productivos que hacia la pobreza. Finalmente, el Consejo de Investigación Agrícola de Bangladesh (BARC, por sus siglas en inglés) es el cuerpo que, desde una posición superior, coordina la investigación y evalúa el proyecto, aunque en la práctica el BARC no tenga una función clara al interior del proyecto debido a su limitada capacidad para monitorear las actividades en el campo.

Durante la década de los ochenta, muchas de las ONG de Bangladesh estuvieron involucradas en la promoción de la acuacultura entre sus grupos organizados de campesinos marginales y sin tierra, proporcionándoles créditos y apoyo técnico. Además de las tres agencias gubernamentales descritas, existen cinco ONG involucradas en el proyecto ICLARM. Para superar las limitaciones del sistema de extensión del gobierno, el proyecto ha hecho la invitación a las ONG para actuar como agentes adicionales de extensión, trabajando de manera conjunta con el DF para distribuir la tecnología a sus propios grupos objetivo (normalmente campesinos sin tierras, especialmente mujeres). Las ONG también han sido invitadas a retroalimentar a los investigadores y capacitadores del FRI respecto a los resultados y las necesidades de la investigación. El personal de campo de las ONG es capacitado por personal del FRI y del ICLARM junto con el DF, de manera que esta capacitación pueda después ser transmitida a los campesinos mediante la demostración y la práctica. Además, las ONG otorgan créditos a los miembros de su grupo. Las ONG involucradas son el Comité para el Avance Rural de Bangladesh (BRAC), Proshika -dos de las más grandes ONG del país-, y tres pequeñas oNG locales: Banchte Shekha y Jagorani Chakra, con sus sedes en Jessore, al 
sur del país; y Thengamara Mohila Sabuj Sangha (TMSS) que realiza sus actividades en Bogra, al norte.

Para el proyecto ICLARM, el desarrollo de vínculos de cooperación entre los investigadores del gobierno y las ONG resulta central en la idea del desarrollo de prácticas de acuacultura de baja inversión y bajo costo, que puedan ser adoptadas y mantenidas por una población rural de bajos ingresos, capaz, a su vez, de retroalimentar a los investigadores a través del personal de campo de las ONG y de los trabajadores de extensión. Cada grupo de las ONG mantiene un banco de datos detallado para sus propósitos. Los supuestos clave son que las ONG necesitan apoyo técnico en sus programas de acuacultura, el cual puede conseguirse a través de la capacitación especializada, y que las ONG tienen una gran fuerza comparativa para establecer vínculos de origen popular (Gupta y Shah, 1992).

\section{Discusión teórica}

El trabajo teórico reciente en las ciencias sociales ha explorado los diferentes tipos de conocimiento y formas de representación incluidas en los proyectos de desarrollo. Siguiendo la perspectiva teórica de Foucault (1971) acerca de las formas en que el conocimiento es histórico, político y socialmente construido como discurso, el antropólogo James Ferguson (1990) demostró cómo un proyecto ganadero financiado por el Banco Mundial en Lesotho necesitó primero construir y representar un problema rural que podría entonces ser resuelto por una intervención del proyecto. Sin embargo, Ferguson argumenta que esta realidad construida o discurso, que se requería para justificar la existencia del proyecto, no necesariamente correspondía a las realidades locales, y de hecho era dirigido por las estructuras más amplias del poder institucional, al interior de las cuales las agencias externas intervenían en la vida económica y social de Lesotho.

Esta perspectiva resulta útil por dos razones: en primer lugar, abre la posibilidad de que exista mucho más detrás de los proyectos de desarrollo de lo que normalmente se describe en la literatura oficial sobre proyectos (que pueden, desde luego, tanto apoyar como restringir sus objetivos oficiales). Si estos discernimientos se hacen más visibles para los actores de los proyectos, es posible que se aprenda más acerca del progreso y potencial de estos últimos. En segundo lugar, sugiere que entre los diferentes actores participantes pueden coexistir múltiples realidades al in- 
terior de un proyecto, cuyo reconocimiento puede ayudar a explicar las diferentes motivaciones para involucrarse en ellos, así como a estimar la probabilidad de que puedan surgir resultados inesperados.

Todo lo anterior indica que la visión convencional -y todavía común- de los proyectos como sistemas lineales y controlados deja de lado aspectos fundamentales de su naturaleza, orígenes y carácter, y que es posible descubrir más información que promueva un nivel más alto de aprendizaje institucional. De manera particular, los intentos de comprender los esfuerzos actuales para negociar entre diferentes intereses y reconciliar conflictos puede ofrecer la clave para un mejor desempeño. En un artículo, Long (1996: 57-58) escribe:

Las interacciones entre el gobierno y las agencias externas involucradas en la instrumentación de programas particulares de desarrollo, y los llamados receptores o población campesina, no pueden entenderse de manera adecuada a través del uso de concepciones generalizadas, como "relaciones estado-campesinado", o recurriendo a conceptos normativos como "participación local". Estas interacciones deben ser analizadas como parte de los procesos actuales de negociación, adaptación y transferencia de significados que tienen lugar entre los actores específicos interesados.

Long demuestra aquí lo que él llama la metodología de “análisis de interfase", pero sus comentarios son igualmente relevantes para la perspectiva procesual que hemos adoptado en este trabajo, y que se discutirá en este documento.

\section{El discurso de cooperación}

Es sólo recientemente que los gobiernos y quienes financian los proyectos han 'descubierto' a las ONG y las han situado en posiciones más prominentes al interior de los proyectos de desarrollo (Edwards y Hulme, 1995). El gobierno de Bangladesh se comprometió de manera explícita a trabajar con las ONG como "socios para el desarrollo" a partir del Quinto Plan de Cinco Años, elaborado en 1990.

Sin embargo, el lenguaje de la cooperación es flexible y, como hemos señalado, puede también ser visto como un discurso foucaultiano producido por la prevaleciente conformación del poder y la influencia institucionales, a medida que las agencias de desarrollo, tanto el gobierno, como las ONG y el Centro Interna- 
cional de Investigación Agrícola (el IARC, por sus siglas en inglés) compiten por recursos y estatus en relación con la dotación externa de éstos. Bangladesh es uno de los países más dependientes de los apoyos a nivel mundial, que suman casi $8 \%$ del PIB. Esto significa que lo que respecta a las cooperaciones, a los vínculos y a otros arreglos de colaboración, puede no ser tan directo como parece, puesto que es probable que éstos estén vinculados a las más amplias negociaciones de los recursos entre los actores de las organizaciones. Por ejemplo, Biggs y Neame (1995) discuten que los modelos lineales del desarrollo tienden a obscurecer el hecho de que las ONG no son agencias individuales, sino que operan en un contexto más amplio basado en negociaciones con redes y coaliciones más amplias, tanto formales como informales, con otras organizaciones. El proceso de negociación puede ser usado por las ONG (y por otras organizaciones) para desafiar las percepciones de quienes otorgan los financiamientos y del gobierno $\mathrm{y}$, por supuesto, para lo contrario.

Dos ejemplos tomados del proyecto ICLARM resultan relevantes:

- Los motivos de las organizaciones para involucrarse en cooperación (en términos de lo que cada una quiere obtener de su colaboración) pueden perfectamente diferir entre las organizaciones participantes. Por ejemplo, mientras que el ICLARM ve a las ONG como las encargadas de difundir las tecnologías de acuacultura entre los campesinos y de proporcionar retroalimentación, la ONG Proshika tiene sus propios programas, por ejemplo, el intento de influir entre los campesinos y los investigadores acerca del uso de tecnologías más orgánicas de acuacultura.

- Los arreglos en la práctica pueden ser diferentes de aquellos descritos de manera oficial. Por ejemplo, mientras que, en teoría, DF tiene autoridad para la extensión de la acuacultura en el país, en la práctica no tiene el personal necesario para llevar a cabo esta labor, pero no necesariamente está dispuesto a delegar esta tarea a las ONG porque éstas compiten por la misma legitimidad y los mismos escasos recursos.

Existen también suposiciones contrarias detrás de la centralidad del Fri en el trabajo del ICLARM en Bangladesh. Aunque es claro que esta relación tiene sus raíces en la autoridad del ICLARM como organización internacional de investigación para establecer vínculos y tratar de fortalecer a la institución nacional de investiga- 
ción 'apropiada' en lo que respecta a la investigación sobre acuacultura, surgen dos problemas de esta estrategia.

El primero se refiere a las dudas que existen en algunos círculos acerca de la efectividad del FRI como institución de investigación y acerca de su forma de operar, tanto en términos de la permanente escasez de recursos, como de la cultura institucional, lo que hace poco probable el viraje hacia una investigación acuícola centrada en los campesinos. ${ }^{4} \mathrm{El}$ segundo problema es la existencia de dudas más amplias acerca de la importancia global de las restricciones técnicas en la acuacultura, que pueden ser resueltas mediante la investigación científica a través de proyectos como éste, comparadas con las restricciones sociales y económicas (Worby, 1994; Lewis, Wood y Gregory, 1996).

Por lo tanto, es tentador sugerir que el ICLARM y el FRI se necesitan mutuamente para la supervivencia institucional individual de cada organización mucho más de lo que las familias campesinas de bajos recursos de Bangladesh necesitan de nuevas tecnologías para la acuacultura. El FRI evidentemente necesita un patrocinador, al igual que muchas de las organizaciones semejantes en Bangladesh. Por otro lado, los campesinos en el ámbito de la aldea que tratan de involucrarse más en la acuacultura están luchando con problemáticas como el acceso a los derechos sobre las lagunas, la dotación oportuna de materiales adecuados para la acuicultura, y con un acceso al crédito y a los mercados que dista mucho de ser el adecuado; estos puntos se abordan detalladamente adelante.

Entre más se descubre acerca de estos discursos, más podemos determinar las bases prácticas para la colaboración, y las limitaciones al interior del proyecto que pudieran distorsionarlo. La investigación ODI sugiere que en algunos casos la base para el vínculo de colaboración fue mal ubicada, mientras que, al mismo tiempo, otras oportunidades de complementariedad entre las organizaciones y los proyectos fueron ocasionalmente desperdiciadas. Por ejemplo, el Proyecto oda de Pesca del Noroeste, un proyecto de investigación y extensión en acuacultura, con muchas similitudes y lecciones que compartir con el proyecto ICLARM (ha establecido vínculos con trece ONG), no tiene vínculos formales con el FRI y actualmente ha cambiado sus objetivos originales de producción e investigación por el de la extensión de tecnologías

\footnotetext{
${ }^{4}$ Comunicación personal, ODA y entrevistas con otras organizaciones en Bangladesh
} 
ya existentes. Tampoco parece haber un nivel particularmente alto de mutuo aprendizaje entre el ICLARM y el ODA en Bangladesh.

Otra característica del efecto potencialmente distorsionador del discurso de la organización dominante es que se vuelve necesario para la acuacultura el ser representada sobre todo como un problema técnico (debido a que los dos socios institucionales clave tienen una prioridad de investigación), aun cuando hay cada vez mayor evidencia proveniente de las investigaciones y de la experiencia de las ONG que resalta el hecho de que las limitaciones para la intensificación de la acuacultura en Bangladesh son principalmente económicas y sociales. Entre estas limitaciones se encuentran la poca disponibilidad de recursos, los conflictos en torno al uso múltiple de las lagunas, las dificultades para el arrendamiento seguro de las lagunas, el alto nivel de riesgo de inversión al que los aldeanos de bajos ingresos se oponen enormemente, las complejidades de género en la distribución del trabajo y la ganancia al interior de las familias, y los asuntos de clase y patronazgo en donde los dueños de las lagunas pueden reclamarlas una vez que quede claro que éstas son provechosas (Worby, 1994; Lewis, Wood y Gregory, 1996). Estas inflexibles realidades contrastan mucho con la imagen oficial de Bangladesh como un país salpicado de cientos de miles de lagunas inutilizadas o subutilizadas con potencial para un incremento masivo de la producción acuícola.

\section{La metodología de investigación}

El proyecto de investigación ODI aseguró su financiamiento gracias a los medios restringidos del ODA; inició en marzo de 1994 y fue calendarizado para llevarse a cabo durante dos años. El plan básico de investigación era que el ODI documentara, en principio, la historia del proyecto ICLARM, con énfasis particular en los procesos de toma de decisiones, en los vínculos de cooperación tanto exitosos como no exitosos, y en las expectativas y percepciones de la organización acerca de las actividades del proyecto. Esto puede semejarse a la idea de la construcción de una "etnografía institucional” del proyecto, término empleado por Escobar (1995) para describir el registro detallado de los procesos y las relaciones utilizando enfoques y metodologías antropológicas. La idea era desarrollar e instrumentar, con los participantes y beneficiarios del proyecto, las 'correcciones de rumbo' que se orientarían a los problemas y a las limitaciones detectadas. 
La intención original del proyecto de investigación y documentación ODI fue conformar tres talleres con participantes del proyecto ICLARM, seguidos de un grupo semiestructurado y de entrevistas individuales. Las discusiones y entrevistas iniciales del taller fueron grabadas para proporcionar un punto de referencia para los supuestos, actitudes y experiencias contra las cuales podía debatirse en las sesiones, los éxitos y fracasos reconocidos, y las soluciones desarrolladas. Las técnicas de observación participante también se emplearían tanto en la oficina del proyecto como en las salidas de campo a las localidades donde las nuevas tecnologías estaban siendo presentadas a los campesinos por las ONG, el gobierno y el personal del proyecto. Además de ello, se consultaría la documentación del proyecto, se trazaría una historia alternativa de éste, incluyendo los resultados planeados e inesperados, así como discusiones comparativas con otras organizaciones involucradas en acuacultura.

El concepto del "proyecto como proceso" fue fundamental para la investigación y apuntaló la selección de una forma de monitorear el proceso para documentar las expectativas y las actividades, y para planear las correcciones de rumbo. La metodología de monitoreo e investigación del proceso -un conjunto de enfoques alternativos al monitoreo convencional, flexibles y en evolución- difiere de lo que en algunas ocasiones se ha denominado la visión 'heliográfica' de los proyectos, que confía en la planeación lineal y en el diseño de los proyectos, a menudo como sistemas cerrados. En contraste, el monitoreo e investigación de los procesos descansa sobre el supuesto de que los proyectos son sistemas abiertos en donde las soluciones a los problemas pueden surgir a través de la experimentación y la práctica, más que a través del diseño. Se concibe al desarrollo como un proceso dinámico que puede ser percibido de diferentes maneras por actores sociales e institucionales diversos, y que probablemente genera importantes resultados inesperados (Mosse, 1998). ${ }^{5}$

${ }^{5}$ El interés actual en concebir los proyectos en términos de proceso tiene paralelo hasta cierto punto en las reflexiones recientes entre los teóricos del cambio organizacional, como Dawson (1994: 4) quien escribe: “...las organizaciones que experimentan una transición deben ser estudiadas 'mientras suceden' de manera que los procesos asociados con el cambio puedan hacerse evidentes a lo largo del tiempo y en su contexto... Este marco temporal de cambio puede también ser empleado para acomodar la existencia de una cantidad de historias diferentes acerca del proceso de transición organizacional... La versión dominante u 'oficial' del cambio puede a menudo reflejar el posicionamiento político de ciertos individuos o grupos clave al interior de una organización, en vez de operar como una verdadera representación de la práctica del manejo de la transición". 


\section{El análisis del proyecto ICLARM como proceso}

Como se ha señalado, el proyecto ICLARM está en realidad conformado por una serie de proyectos orientados al desarrollo y a la introducción de tecnologías sustentables de acuacultura. Estos proyectos se han extendido y ajustado en la medida en que la experiencia (y los fondos disponibles) lo han permitido. En ese sentido, puede ser visto como punto de partida tanto para la comprensión como para el acercamiento a una serie de problemas importantes en torno a la acuacultura y, de manera más general, a la cooperación interorganizacional. Los problemas y los resultados no esperados, como se discutirá aquí, pueden resultar valiosos y deben, por lo tanto, ser registrados más que omitidos en los documentos de los proyectos. Esta es una de las ventajas de emplear este tipo de registro del proceso.

Gran parte del proyecto ODI se destinó a discutir las intenciones originales del proyecto ICLARM y a compararlas con lo que en realidad estaba ocurriendo en la práctica. Los vínculos de cooperación al interior del proyecto se categorizaron y se identificaron varios niveles de cooperación. Mediante entrevistas con personas clave del proyecto se hicieron intentos (algunos de los cuales quedaron inconclusos, como se explicará en la sección 7) para entender cómo habían funcionado estos vínculos, las limitaciones que se enfrentaron y las posibles formas en que éstas pudieron superarse.

Algunos de estos vínculos resultaron eficaces, otros débiles; estos vínculos se clasifican de manera preliminar en el cuadro 1. En el caso de aquellos vínculos que fueron categorizados como débiles, se discutieron algunas acciones para corregirlos. Por ejemplo, cuando se identificaron tensiones entre las grandes ONG y las pequeñas, y entre éstas y el personal local de DF, se exploró la posibilidad de fortalecer, a través de cabildeos y negociaciones, un foro regional de acuacultura de la Asociación de Organizaciones para el Desarrollo en Bangladesh (ADAB, por sus siglas en inglés), aunque con resultados diversos. Sin embargo, cada uno de estos vínculos y los subsecuentes intentos por corregir el rumbo ayudaron a entender mejor el problema global de la cooperación. 


\section{Cuadro 1}

\section{Puntaje en vínculos de cooperación y su eficacia relativa}

\begin{tabular}{|l|l|l|}
\hline \multicolumn{1}{|c|}{ Vínculo } & \multicolumn{1}{|c|}{ Mecanismo } & Efectividad \\
\hline Campesinos con campesinos & Contactos informales & M \\
& Sesiones demostrativas & F \\
División familiar del trabajo & M \\
\hline $\begin{array}{l}\text { Campesinos con personal de } \\
\text { campo de ONG }\end{array}$ & Sesiones de capacitación & M \\
Sesiones demostrativas & F \\
\hline $\begin{array}{l}\text { Campesinos con personal de } \\
\text { extensión de DF }\end{array}$ & Reuniones regulares de ONG & F \\
\hline $\begin{array}{l}\text { Campesinos con } \\
\text { investigadores }\end{array}$ & Visitas de campo del FRI & D \\
\hline $\begin{array}{l}\text { Trabajadores de ONG con } \\
\text { personal de extensión de DF }\end{array}$ & $\begin{array}{l}\text { Reuniones mensuales (vía } \\
\text { retroalimentación de la ONG) }\end{array}$ & Froyecto \\
\hline $\begin{array}{l}\text { Personal de las ONG con } \\
\text { investigadores del FRI }\end{array}$ & $\begin{array}{l}\text { Sesiones de capacitación del } \\
\text { FRI }\end{array}$ & M \\
\hline $\begin{array}{l}\text { Grandes ONG con pequeñas } \\
\text { ONG }\end{array}$ & $\begin{array}{l}\text { Reuniones de } \\
\text { proyecto } \\
\text { Feunionsuales de } \\
\text { proyecto }\end{array}$ & M \\
\hline
\end{tabular}

Clave: F, fuerte; M, media o variada; D, débil. Nota: Estas son valoraciones indicativas basadas en entrevistas, observación de campo limitada y percepciones de los actores.

Aunque el proyecto ODI no duró lo suficiente como para alcanzar el resultado esperado, se detectaron hallazgos significativos. El proyecto ICLARM había logrado muchos de sus objetivos, los cuales proporcionarán oportunidades a las ONG para acceder al apoyo técnico en sus programas de acuacultura, para reportar los problemas enfrentados por los campesinos con los que trabajan y para comenzar a crear, por primera vez, vínculos con organismos gubernamentales de acuacultura. Para finales de 1994, un total de 3, 563 campesinos (de los cuales 2, 029 eran mujeres) había sido capacitado, 900 lagunas habían sido cultivadas y la tecnología resultó a todas luces efectiva cuando se aplicó 'adecuadamente'. 
Se ha fortalecido de manera particular la curva de retroalimentación de los campesinos hacia los investigadores, a través de las ONG. Se han realizado modificaciones al paquete original de tecnología uniforme del proyecto ICLARM, que ha sido rediseñado en opciones diferentes de manera que se tomen en cuenta las diversas prioridades agroecológicas con base en la retroalimentación proveniente de los campesinos, vía las ONG que participan en diferentes regiones agro-climáticas (ICLARM, 1994). Además, los investigadores del gobierno y de las ONG están, tal vez por primera vez, dialogando entre sí acerca de la acuacultura. En el papel, se ha cumplido en gran medida con los objetivos propuestos. Pero una vez que echamos un vistazo al proyecto tal y como se inserta en un sistema más amplio de relaciones y discursos, queda claro que en ciertas áreas clave serían deseables otros resultados. Como era de esperarse, hay aún algunas áreas débiles:

- Los vínculos entre las organizaciones pueden no durar más allá del término del apoyo del 'respaldo técnico', el trabajo motivador y la dotación de fondos.

- La 'cultura institucional' al interior de las agencias gubernamentales, como el FRI, continúa en lo fundamental orientada alrededor del viejo modelo de transferencia de tecnología. El lenguaje participativo se está asimilando mucho más rápido de lo que se está llevando cabo y las actitudes de algunos de los nuevos miembros se están volviendo más abiertas, pero esto tiene poca influencia si no cuentan con el apoyo de sus superiores.

- Las relaciones entre las ONG nacionales y las locales son a menudo débiles y faltas de coordinación. Las grandes ONG tienden a concentrar muchos recursos y requieren de poco apoyo técnico, salvo el más especializado; las oNG más pequeñas necesitan tanto recursos, como apoyo y capacitación técnica básica. Los intentos por fortalecer los vínculos a través del foro ADAB de acuacultura, creado por la organización nacional que agrupa a las $\mathrm{ONG}$, son cuando mucho tentativos, en parte debido a que los intereses de las ONG más grandes tienden a prevalecer al interior de $\mathrm{ADAB}$ por encima de aquellos de las ONG más pequeñas, y muchas de las ONG pequeñas no se afilian a ADAB y quedan, por lo tanto, excluidas de cualquier beneficio que pudiera surgir. ${ }^{6}$

${ }^{6}$ Véase Noble (1995) para una descripción de la iniciativa ADAB y los problemas enfrentados en la cooperación ONG-ONG en acuacultura. Noble hace resaltar el hecho de que no existe aún un proyecto de colaboración formal entre ONG involucradas en acuacultura. Una excepción a esta generalizada carencia de colaboración es Caritas, que sí proporciona apoyo técnico informal a pequeñas oNG locales. 
- Ha existido un nivel relativamente bajo de coordinación, así como de intercambio de lecciones aprendidas entre el ICLARM y el ODA, que opera un proyecto más grande en Bangladesh, con al menos algunos intereses comunes.

- Es posible que los campesinos sean vistos ahora por algunos organismos gubernamentales como capaces de probar y adaptar las tecnologías que se les proporcionan, pero no todavía como una fuerza capaz de orientar de manera significativa los programas de investigación.

- Los problemas enfrentados por los campesinos de bajos ingresos en la adopción sustentable de tecnologías de acuacultura son todavía agudos, y sobre todo de carácter social y económico, más que técnico.

Sin embargo, a pesar de lo grave de dichos problemas, éstos conforman un programa para la acción de las organizaciones y de los proyectos que intentan combatirlos. Por lo tanto, es posible generar una agenda para la acción práctica al crear o expandir el 'espacio de acción' a través del enfoque procesual adoptado por la investigación ODI.

\section{Problemas emergentes}

Este análisis desembocó en varias iniciativas, desarrolladas ya sea por el proyecto ICLARM o por el proyecto ODI (o de manera conjunta), para profundizar en el aprendizaje de los proyectos y llevar a cabo correcciones de rumbo. Los principales problemas que surgieron están sintetizados en este apartado.

En primer lugar, las contingencias del registro de los proyectos no necesariamente llevan a la interrogación de toda la evidencia disponible (disponible para los socios del proyecto) relacionada con el desempeño de la cooperación o con la internalización de las lecciones aprendidas. El hecho de que exista un tercer actor involucrado -el ODI, en este caso- puede jugar un papel útil al respecto. En segundo lugar, la cooperación sólo tiene sentido si demuestra haber logrado ciertos resultados que no hubieran sido posibles por cada uno de los socios de manera aislada. Algunas veces la retórica o discurso de cooperación puede usarse en sí para exigir atención y recursos, y esto puede responder a una gran variedad de intereses y objetivos políticos. La documentación del proceso puede alentar a los participantes del proyecto a confrontar las brechas entre lo que están diciendo, lo que dicen estar haciendo, y lo que en realidad hacen. 
En tercer lugar, el proceso de construcción de la etnografía institucional de un proyecto puede generar actividades nuevas y suplementarias orientadas a corregir las limitaciones. Algunas veces estas actividades surgen de los resultados inesperados. Pero muchas organizaciones inmersas en un entorno de dependencia de recursos tienen dificultades para enfrentarse a las preconcepciones y a las envidias entre organizaciones, así como para mejorar la comunicación y, por lo tanto, el desempeño al interior del proyecto. Esta es una de las razones por las que el ejercicio de investigación de ODI terminó prematuramente. Sin embargo, el inicio de los aportes de las ONG en la formulación de una nueva política pesquera constituye un valioso resultado inesperado. Igualmente, hemos visto que las organizaciones decidieron, después del primer taller, asumir un papel más activo en el fortalecimiento del nuevo (y en algunos aspectos inactivo y marginado) foro de ONG de acuacultura.

Se está desarrollando un conjunto de líneas de acción a futuro, a través de las cuales los proyectos pueden explorar en profundidad los problemas de la cooperación, y que permiten a ésta ser valorada con base en los resultados. Se espera que estas líneas de acción, que contienen las experiencias surgidas de este proyecto, sean de valor para el ICLARM y el ODA al planear e instrumentar futuros proyectos (Lewis y Ehsan, 1996).

Finalmente, existe sobre todo una visión de transferencia de tecnología en muchas de las organizaciones, particularmente en las gubernamentales. Como se ha señalado, no es evidente el hecho de que las limitaciones tecnológicas sean importantes en el desarrollo de la acuacultura. ${ }^{7}$ Sin embargo las modificaciones a la cultura institucional de las organizaciones gubernamentales (en particular), así como de las ONG, que las hagan ser más receptivas a las necesidades de los campesinos y perseguir estrategias de trabajo más flexibles a través de la cooperación, requerirán soluciones a largo plazo. Sin embargo, esta investigación-acción está comenzando a demostrar que los talleres interproyectos e interorganizacionales, así como las discusiones con un tercer actor pueden resultar creativos para orientar la cultura de las organizaciones hacia esa meta.

\footnotetext{
${ }^{7}$ Sin embargo, existen experiencias que apuntan al hecho de que una vez que los campesinos están convencidos del valor de la tecnología, requieren de poco apoyo por parte del personal de extensión. Por ejemplo, el éxito de la difusión de la cultura de desmenuzado de pescado ha impulsado a Gregory y Kamp (1996: 21-22) a escribir que "una tecnología de la cual realmente vale la pena su difusión no es difícil de difundir".
} 


\section{Reflexiones en torno a los problemas metodológicos}

Durante el transcurso de la investigación oDI fue evidente que habíamos subestimado las dificultades metodológicas que podíamos encontrar a lo largo de la investigación y la documentación por parte de un tercer actor. Hubo varios factores durante el trabajo que es preciso delinear:

- Bangladesh es un país en donde las organizaciones e instituciones locales, tanto ONG como gubernamentales, compiten por los financiamientos y la credibilidad en un contexto altamente dependiente de los recursos. Se construyen alianzas entre organizaciones individuales, y éstas pueden, en algunas ocasiones, adoptar el carácter de relaciones patrón-cliente. Sin importar que podamos describirlas, estas relaciones son altamente sensibles, y por lo tanto es probable que se resistan a los esfuerzos externos por documentarlas, esfuerzos que pueden ser percibidos como interferencias. De hecho, esta documentación puede exacerbar las tensiones al interior del proyecto. ${ }^{8}$

- El conjunto de problemas antes señalados no se limita exclusivamente a las organizaciones locales o nacionales. Las organizaciones internacionales de investigación, como el ICLARM, están bajo una presión externa muy fuerte por parte de quienes las financian para demostrar la centralidad de sus programas de investigación dentro de las prioridades de la política nacional, y pueden estar, comprensiblemente, más preparadas para documentar las fortalezas que las debilidades de sus programas. Las tensiones entre las oficinas centrales y el personal de campo fueron percibidas en términos de grados variables de identificación con la investigación ODI. Esto resultó verdadero tanto en términos espaciales, dada la distancia entre las oficinas centrales del ICLARM y la oficina del proyecto Dhaka, como en términos temporales, en donde algunos de los acuerdos iniciales del proyecto se perdieron cuando uno de los miembros más antiguos del personal del ICLARM y codiseñador del proyecto de investigación ODI, se fue.

\footnotetext{
${ }^{8}$ Un problema persistente fue la sensibilidad del gobierno de Bangladesh en torno al procedimiento y el control, cuando se tratara con organizaciones externas. Una debilidad clave de la investigación oDI fue que no había sido incluida como parte de la propuesta del proyecto oficial y original del ICLARM, sino que fue un agregado que no encajaba en ninguna categoría burocrática precisa.
} 
- Como parte del impulso entre los actores clave del proyecto para asegurar la supervivencia institucional de ambas organizaciones, y los vínculos institucionales que mantenían unido el proyecto, hubo una tendencia en el proyecto ICLARM a percibir las perspectivas de la investigación ODI como si ya hubieran sido comprendidas y puestas en marcha. Desde luego, esto era cierto en muchos casos, pero el deseo por parte del proyecto de 'poseer' tanto sus problemas como sus logros dificultó el trabajo conjunto con los actores del proyecto para diseñar y poner en práctica los adecuados ajustes.

- Otros problemas giraban en torno a las diferentes versiones en conflicto de la realidad; las tensiones entre los investigadores internos y externos, y el personal del proyecto; cuestiones de estatus entre los investigadores nuevos y los miembros más antiguos del personal; tensiones entre los investigadores y los investigados; y la dificultad generalizada (y comprensible) que la mayor parte de la gente experimenta para hablar de 'lo que está mal'.

Estos problemas llevaron eventualmente a una situación en la que la metodología prevista era incapaz de considerar las complejidades políticas de los proyectos inter-organizacionales en Bangladesh, por no hablar de las sutilezas de las cuestiones fundamentales de la investigación. La resistencia al proyecto de documentación ODI por parte de algunos actores del proyecto cobró la forma de negociación y conflicto acerca de la orientación que debía tomar la investigación; la falta ocasional de cooperación para presentar a los investigadores con poseedores de información clave, quienes agilizarían el proceso; el cuestionamiento de la legitimidad de las sugerencias de ODI para posibles 'correcciones de rumbo'; la sugerencia de que la investigación oDI se había desviado de sus objetivos originales; y finalmente, la exigencia de que el proyecto terminara antes de lo previsto debido al peligro de las actividades del proyecto, y a que la legitimidad de algunos actores clave podría verse amenazada.

Como resultado de estas dificultades, sólo el primero de tres talleres proyectados se llevó a cabo y sirvió para presentar los objetivos del proyecto ODI y para destacar los logros y los problemas en proceso. Este taller cumplió su propósito al generar una gran cantidad de información básica a la que se dio continuidad a través de varias estancias en campo y de entrevistas 
individuales semidirigidas con actores del proyecto ICLARM. Sin embargo, continuó existiendo una inquietud fundamental entre algunos de los miembros del personal respecto a que la presencia de extraños (lo que no se había indicado en los documentos del proyecto original aceptado por el ICLARM y por el Gobierno de Bangladesh) podría desestabilizar las precarias relaciones entre las diferentes organizaciones gubernamentales, los patrocinadores y las ONG. Esta preocupación fue hasta cierto punto confirmada, y en más de una ocasión ciertos actores utilizaron información imprecisa para propósitos políticos, lo que generó una carga extra para los directores de los proyectos.

Las dificultades experimentadas en el despliegue de esta metodología de investigación y monitoreo del proceso, y de hecho, el fracaso parcial del proyecto de documentación en sí mismo contiene, sin embargo, varias visiones útiles acerca de:

- El trabajo de los proyectos interorganizacionales en Bangladesh en el escenario de la competencia por recursos escasos.

- El discurso de cooperación y las formas en que éste se traduce en la práctica.

- Las formas en que los problemas de la acuacultura han sido construidos en Bangladesh por parte de las organizaciones de desarrollo junto con sus soluciones.

- Las formas a través de las cuales la retórica de la participación puede ser asimilada de una manera relativamente fácil con una transferencia de tecnología del modelo tecnológico.

De esta manera, las tensiones generadas por la metodología de investigación crearon una serie de problemas prácticos muy reales; pero al mismo tiempo han ayudado a producir algunas visiones valiosas al interior de los procesos de un proyecto. Pocos de estos problemas son tocados en la literatura oficial sobre proyectos, pero pueden ser provechosamente explorados en trabajos adicionales realizados específicamente con ese propósito, como el tipo de monitoreo de procesos que hemos intentado aquí. Como escribe Escobar (1995)

...un discurso textualmente mediado sustituye a las relaciones y prácticas reales de los "beneficiarios", enterrando su experiencia en la matriz que organiza la representación de la institución. 
En cierto nivel este enfoque es sostenido por el presente trabajo en lo que toca a que el proyecto tiende a demandar y a representar un nivel de participación que rebasa con mucho las relaciones reales que existen, tanto con las personas en los márgenes, como en el nivel institucional entre las diferentes organizaciones participantes. Pero hemos tratado aquí de rebasar eso; a diferencia de Escobar, quien presenta una imagen esencialmente pesimista, creemos que el discurso dominante no es monolítico, sino que puede contener algunas oportunidades para la "libertad de acción" mediante el mejoramiento del espacio para la negociación y la transparencia (Gardner y Lewis, 1996).

En términos más prácticos, la principal lección que surge es que existe la necesidad de sensibilizarse al conducir la investigación y la documentación de los procesos, particularmente en lo que concierne al papel del agente o agentes externos que llevan a cabo el monitoreo. Este agente externo requiere un amplio conjunto de habilidades en esta área, como la habilidad de generar confianza entre todos los sectores del proyecto y entre los diversos participantes de las organizaciones, manifestando un conocimiento de las, a menudo, inevitables contradicciones implicadas por las diferentes perspectivas de los actores, mostrando respeto por el duro trabajo realizado por muchos de los miembros del personal involucrado. En el caso de la presente investigación, no fuimos completamente exitosos en asegurar que estas habilidades se hayan puesto siempre en práctica.

\section{Conclusiones}

A pesar de los problemas prácticos enfrentados en esta investigación, puede decirse que la metodología de investigación y documentación del proceso desarrollada durante la misma ha generado una serie de enfoques útiles acerca de los trabajos al interior de los proyectos de desarrollo, y de los campos de poder y discurso por los que éstos están rodeados. En un nivel práctico la investigación también proporciona algunas pistas importantes para el potencial progreso futuro en torno, tanto a la acuacultura en Bangladesh como, en un contexto más amplio, a la cooperación interorganizacional. Si bien es difícil generar y mantener una cooperación "proactiva" en un contexto de dependencia de recursos como Bangladesh, y si bien los diferentes análisis y prescripciones para promover el desarrollo acuícola están también en competencia unos con otros, puede haber espacios significativos 
de libertad de acción. Algunas organizaciones empiezan a enfrentar ciertos problemas para lograr que las familias económicamente marginales mejoren sus ingresos y su alimentación.

Sin embargo, el problema de la dependencia no sólo significa que la sustentabilidad de los vínculos entre las organizaciones es cuestionable, sino que también atrae la atención sobre la naturaleza y el énfasis en la receta tecnológica ofrecida por muchos de los proyectos de este tipo en Bangladesh. Esto es debido a que el flujo de recursos puede ayudar a determinar la forma en que los problemas se construyen, de la misma manera en que ayuda a estructurar la forma en que se realizan las intervenciones. El enfoque de documentación del proceso empleado en esta investigación ayuda a mitigar el funcionamiento de estas fuerzas mayores en el contexto de la acuacultura.

Existen decisiones difíciles a futuro para los encargados del desarrollo de la acuacultura en Bangladesh. Las iniciativas de investigación y extensión necesitan volverse más participativas, menos verticales para, de manera ideal, superar las estrechas definiciones de la retroalimentación por parte de los campesinos y dar paso a modelos en los que éstos puedan realmente influir en los programas de investigación, no sólo hacer comentarios acerca de las tecnologías que se les ofrecen. Existe también la necesidad de desarrollar organizaciones para aproximarse al espinoso punto de las importantes limitaciones económicas y sociales, de manera que éstas trasciendan la justificación de las supuestas habilidades de las ONG para orientarse a los campesinos, como parte de lo que podría llamarse la perspectiva "instrumentalista" del gobierno y de las relaciones entre ONG. Al llevar a cabo un mayor proceso de documentación del tipo que se describe aquí, se espera que el desequilibrio que probablemente se genere pueda mantenerse más en el camino de la creatividad y de las soluciones, que en el de la destructividad y la contradicción. La cooperación activa entre las ONG, y el gobierno y las IARC puede aún revelar un mayor potencial. 


\section{Bibliografía}

Biggs, S. y A. Neame (1995), "Negotiating room for manoeuvre: reflections concerning NGO autonomy and accountability within the new policy agenda", en M. Edwardes y D. Hulme (eds.), Non-governmental organizations - performance and accountability: beyond the magic bullet, Earthscan, London.

Dawson, P. (1994), Organizational change: a processual approach, Paul Chapman, London.

Escobar, A. (1995), Encountering development: the making and unmaking of the third world, Princeton University Press, Princeton, New Jersey.

Farrington, J. y D. J. Lewis con S. Satish and A. Miclat-Teves (1993), (eds.), NGOs and the State in Asia: rethinking roles in sustainable agricultural development, Routledge, London.

Ferguson, J. (1990), The anti-politics machine: "development", depoliticization and bureaucratic power in Lesotho, Cambridge University Press, Cambridge.

Foucault, M. (1971), “The order of discourse”, en R. Young (ed.), Untying the text: a post-structuralist reader, Routledge and Kegan Paul, London.

Gardner, K. y D. J. Lewis (1996), Anthropology, development and the post-modern challenge, Pluto, London.

Gregory, R. y K. Kamp (1996), Aquaculture extension in Bangladesh: experiences from the northwest fisheries extension project 1989-92, ODA BAFRU/Department of Fisheries, Dhaka.

Gupta, M. V. y M. S. Shah (1992), NGO linkages in developing aquaculture as a sustainable farming activity - A case study from Bangladesh, documento presentado en Asian Fanning Systems Symposium, Colombo. 
ICLARM (1994), Proceedings of the workshop on technology transfer through NGOs and feedback to research, Workshop notes, 20-21 de marzo.

Lewis, D. J., G. D. Wood y R. Gregory (1996), Trading the silver seed: local knowledge and market moralities in aquacultural development, Intermediate Technology Publications and Dhaka, University Press Limited, London.

Lewis, D. y K. Ehsan (1996), Guidelines towards promoting 'active' inter-agency partnerships in sustainable development: lessons from an aquaculture project in Bangladesh, ODI/ ICLARM reporte al Overseas Development Administration, London.

Long, N. (1996), "Globalization and localization: new challenges to rural research", en Henrietta L. Moore (ed.), The future of anthropological knowledge, Routledge, London.

Mosse, D. (1996), Process monitoring and process documentation: evolving methods for social research and development practice - cases and issues, First draft, Centre for Development Studies, University of Swansea.

Mosse, D., J. Farrington y A. Rew (eds.) (1998), Development as process: concepts and methods for working with complexity, Routledge, London.

Noble, F. (1995), "NGOs and the aquaculture and fisheries forum of ADAB", in W. A. Shah (ed.), Pond fisheries in Bangladesh, Environment and Resources Analysis Centre, Dhaka.

Worby, E. (1994), Hitting hairs and splitting targets: anthropological perspectives on fish culture technology transfer through NGOs in Bangladesh, documento de conferencia de Rockefeller Foundation, Addis Ababa.

Enviado: 19 de julio de 2000 Aceptado: 31 de enero de 2001 